\title{
DIFFERENTIAL INTERFEROMETRY IMAGING
}

\author{
R. G. PETROV, S. LAGARDE AND M. N'GUYEN VAN KY \\ Département d'Astrophysique \\ Université de Nice - Sophia Antipolis \\ U.R.A.709 du C.N.R.S., Parc Valrose \\ F-06108 Nice Cedex, France
}

\begin{abstract}
Differential Interferometry (DI) combines high spectral and high spatial resolution. On non resolved objects, it yields the angular variation $\vec{\varepsilon}(\lambda)$ of the source photocenter as a function of wavelength which has been shown theoretically and experimentally to complement very usefully both interferometric and spectroscopic data in a large number of astrophysical problems. This paper presents the general characteristics of DI which are likely to allow improvements of the Doppler images of stellar surface structures as soon as interferometers with large apertures and baselines approaching $100 \mathrm{~m}$ are available.
\end{abstract}

\section{Introduction}

The present symposium (IAU S-176) has been an excellent illustration of the spectacular successes of Doppler Imaging in the mapping of stellar surface structures. But it gave also an occasion to review some of its difficulties and limitations (Rice 1996, Unruh 1996).

Even in the ideal case, a feature of latitude $\delta$ on a star with inclination $i$ cannot be discriminated from a feature of latitude $-\delta$ on a star with inclination $\pi-i$. This north-south ambiguity is a real burden on stars with inclinations close to $90^{\circ}$, where the possibility to shift any part of a feature at latitude $\delta$ to the latitude $-\delta$ results in a so called mirror effect which can be seen or suspected in numerous Doppler images. Imperfect knowledge of the local line profile can introduce artefacts and has been suspected to be at least partially responsible for the polar caps present in a majority of Doppler images (Strassmeier 1996). Another source of ambiguity can be a 
bad modelisation of the radial velocity field. Errors can be introduced by differential rotation or by non radial oscillations. And, of course, starspots evolving faster than the rotation period are a problem.

For most of the targets of Doppler Imaging, it will be necessary to wait quite a long time before the availability of direct images from phase coherent interferometry because a direct resolution of all but the large structures on the brightest stars needs multi kilometric baselines and a good u-v coverage implying a very large number of telescopes or a very long observing time.

Differential Interferometry (DI) which combines high angular resolution interferometry with medium to high resolution spectroscopy and can be applied to unresolved sources will permit to test and to improve Doppler images much sooner. In the following we present this technique with an emphasis on the characteristics which are likely to be useful for Doppler Imaging. Then we describe the DI data which will be produced by a rotating spotted star and review how it may be used to relax the constraints necessary for a good monitoring of stellar surface structures. Finally we very briefly compare the SNRs of DI and of spectroscopy and conclude with an estimation of the time scale for the first application of Differential Interferometry Imaging.

\section{Differential Interferometry}

\subsection{PRINCIPLES AND HISTORY OF DIFFERENTIAL INTERFEROMETRY}

Differential Speckle Interferometry (DSI) is the application of the high angular resolution speckle interferometric techniques to an astronomical candidate observed simultaneously in different wavelengths or polarisations. It is based on two simple ideas due to Beckers (1982): the shift between two identical speckle patterns can be measured with a precision much higher than the speckle size, i.e. than the diffraction resolution limit and, for a non resolved object, the variation in the speckle positions between two spectral channels is equal to the difference between the source photocenters at the two central wavelengths. Beckers' (1982) list of potential applications already contained Ap stars and the study of their magnetic line variability. Experimental tests (Aime et al. 1984) and SNR estimations (Petrov et al. 1986 , Chelli \& Petrov 1995) confirmed that DI is applicable to a fair number of sources. This was confirmed by the first experimental results on the double star Capella (Lagarde 1994, Petrov et al. 1995) and on the slowly rotating K giant Aldebaran (Petrov et al. 1996) who demonstrated the feasibility of the technique and its huge scientific potential but also illustrated the practical difficulties resulting mainly from the necessity to measure displacements sometimes smaller than $1 / 100$ pixels with intensified cameras 
with relatively large defects and instabilities. Nevertheless, Lagarde (1994) demonstrated theoritically and with computer simulations that it is always possible to reach the theoretical limit of performance of DSI set by photon and speckle noise and a dedicated instrument confirmed this experimentally. Differential Interferometry (DI) is the generalisation of DSI to long baseline interferometers (Petrov 1989).

\subsection{BASIC EQUATIONS OF DIFFERENTIAL INTERFEROMETRY}

Differential interferometry is based on the cross analysis of series of speckle patterns recorded in different spectral channels. If $i(\vec{r}, \lambda)$ describes the instantaneous speckle pattern produced by a source with brightness distribution $o(\vec{r}, \lambda)$ we have:

$$
i(\vec{r}, \lambda)=o(\vec{r}, \lambda) * a(\vec{r})
$$

where $a(\vec{r})$ represents the instrument + atmosphere instantaneous point spread function for the angular coordinate $\vec{r}$ and $*$ stands for convolution. The key condition that $a(\vec{r})$ is the same for all spectral channels can be realised if the overall bandwidth is small enough (typically $10 \mathrm{~nm}$ ) and if the distortions of the optics and the detector are correctly measured and compensated. The data processing is based on the estimation of the average cross spectrum $W\left(\vec{u}, \lambda_{1}, \lambda_{r}\right)$ between a narrow bandwidth $\lambda$ channel and a relatively larger bandwidth reference channel with central wavelength $\lambda_{r}$. If $F(\vec{u})$ represents the Fourier transform of the function $f(\vec{r})$, we can write:

$$
\begin{aligned}
W\left(\vec{u}, \lambda_{1}, \lambda_{r}\right) & =\left\langle O\left(\vec{u}, \lambda_{1}\right) A(\vec{u}) O^{*}\left(\vec{u}, \lambda_{r}\right) A^{*}(\vec{u})\right\rangle= \\
& =O\left(\vec{u}, \lambda_{1}\right) O^{*}\left(\vec{u}, \lambda_{r}\right)\left\langle|A(\vec{u})|^{2}\right\rangle
\end{aligned}
$$

where $O *$ is the complex conjugate of $O$ and $\langle>$ is a time average. Since $<|A(\vec{u})|^{2}>$ is real and positive, the phase $\phi\left(\vec{u}, \lambda_{1}, \lambda_{r}\right)$ of $W\left(\vec{u}, \lambda_{1}, \lambda_{r}\right)$ is this of the cross spectrum of the object brightness distributions, independently from any seeing variation. For a non resolved object it is very easy to show (Petrov 1989) that $\phi\left(\vec{u}, \lambda_{1}, \lambda_{r}\right)$ is proportional to the displacement of the source photocenter between $\lambda_{1}$ and $\lambda_{r}$ :

$$
\phi\left(\vec{u}, \lambda_{1}, \lambda_{r}\right)=2 \pi \vec{u}\left[\vec{\varepsilon}\left(\lambda_{1}\right)-\vec{\varepsilon}\left(\lambda_{r}\right)\right]
$$

where the angular vector $\vec{\varepsilon}(\lambda)$ represents the center of gravity of the brightness distribution $o(\vec{r}, \lambda)$ given by:

$$
\vec{\varepsilon}(\lambda)=\frac{\int \vec{r} o(\vec{r}, \lambda) d \vec{r}}{\int o(\vec{r}, \lambda) d \vec{r}}=\frac{\vec{m}_{1}(\lambda)}{s(\lambda)}
$$

In the following we shall arbitrary set the origin of the angular plane in order to have $\vec{\varepsilon}\left(\lambda_{r}\right)=0$ and we will consider that DI yields $\vec{\varepsilon}(\lambda)$. 
It is important to note the similarity between $\vec{\varepsilon}(\lambda)$ and the spectrum $s(\lambda)$ : both result from integrals of the brightness distribution, but with a different angular weighting. More precisely, $s(\lambda)$ is the zero order moment of $o(\vec{r}, \lambda)$ and $\vec{\varepsilon}(\lambda)$ yields its first order moments $m_{1 x}(\lambda)$ and $m_{1 y}(\lambda)$, components of the angular vector $\vec{m}_{1}(\lambda)$ defined by equation (4). This explains why most of the methods for the interpretation of $\vec{\varepsilon}(\lambda)$ have to be derived from spectroscopy even if it is measured using speckle interferometric techniques. It also indicates that DI is applicable to any astronomical candidates showing spectral features which might result from (relatively) large scale brightness or velocity structures. Then, the angular vector $\vec{\varepsilon}(\lambda)$ will provide the angular scale and the position angle of the source together with new constraints on its model, generally by making it possible to separate the contributions of the local line profile and of the global radial velocity $v(\vec{r})$.

For example, if we assume that we would measure the same spectrum $p(\lambda)$ for all the parts of the star if they had a zero radial velocity, we can write:

$$
o(\vec{r}, \lambda)=b(\vec{r}) p\left[\lambda-\lambda_{0} v(\vec{r}) / c\right]
$$

where $b(\vec{r})$ is the continuum $(p(\lambda)=1)$ brightness distribution and we assume that $v(\vec{r})<<c$ and $\lambda-\lambda_{0}<<\lambda_{0}$. Then, the spectrum is the convolution

$$
s(\lambda)=h_{0}(\lambda) * p(\lambda)
$$

between the local profile and the velocity distribution $h_{0}(\lambda)$. The lattest is equal to the spectrum which would be obtained if $p(\lambda)$ was the $\delta$ function $\delta\left(\lambda-\lambda_{0}\right)$ :

$$
h_{0}(\lambda)=\int b(\vec{r}) \delta\left(\lambda-\lambda_{0}-\lambda_{0} \frac{v(\vec{r})}{c}\right) d \vec{r}=\int_{\mathrm{C}\left(c\left(\lambda-\lambda_{0}\right) / \lambda_{0}\right)} b(\vec{r}) d \vec{r}
$$

where $\mathrm{C}(v)$ represents the ensemble of points with radial velocity $\mathrm{v}$.

The distribution $h_{0}(\lambda)$ is a velocity density: $h_{0}(\lambda) d \lambda$ represents the proportion of the stellar flux emitted by points with a radial velocity between $v(\vec{r})=c\left(\lambda-\lambda_{0}\right) / \lambda_{0}$ and $v(\vec{r})=c\left(\lambda+d \lambda-\lambda_{0}\right) / \lambda_{0}$. If the relation between radial velocity and space is known, as for example for a star rotating as a solid where $v(\vec{r})=V \operatorname{sini} . x / R$ (x being the angular distance to the projected rotation axis and $\mathrm{R}$ the stellar radius), $h_{0}(\lambda) d \lambda$ gives the fraction of the stellar flux emitted in the strip parallel to the rotation axis and located between $x=(R . c / V \operatorname{sini})\left(\lambda-\lambda_{0}\right) / \lambda_{0}$ and $x=(R . c / V \sin i)\left(\lambda+d \lambda-\lambda_{0}\right) / \lambda_{0}$ which just means that $h_{0}(\lambda)$ is a one dimensional image of the star, integrated in the direction of the rotation axis (but with unknown angular scale). This is at the origin of Doppler Imaging even if the independence of $p(\lambda)$ from space is now often replaced by a modelisation of its variation 
with temperature and latitude. Equation (6) shows that if $p(\lambda)$ is unknown, it might be difficult to obtain $h_{0}(\lambda)$. One of the interesting features of DI is that this problem can be solved by combining $s(\lambda)$ and $\vec{m}_{1}(\lambda)$. By analogy with eq. (6), it is easy to write:

$$
\vec{m}_{1}(\lambda)=\vec{h}_{1}(\lambda) * p(\lambda)
$$

where

$$
\vec{h}_{1}(\lambda)=\vec{r} b(\vec{r}) \delta\left(\lambda-\lambda_{0}-\lambda_{0} \frac{v(\vec{r})}{c}\right) d \vec{r}
$$

is the moment of the points in the ensemble $\mathrm{C}$ weighted by their brightness. From a combination of Eqs. (6) and (8), we can built a quantity which is independent from $p(\lambda)$. For example, by analogy with Eq. (2) we can move to the wave number space, compute the Fourier transforms $S(\sigma)$ and $\vec{M}_{1}(\sigma)$ of $s(\lambda)$ and $\vec{m}_{1}(\lambda)$ and built a cross spectrum

$$
S^{*}(\sigma) \vec{M}_{1}(\sigma)=H_{0}^{*}(\sigma) \vec{H}_{1}(\sigma)|P(\sigma)|^{2}
$$

whose phase $\varphi(\sigma)$ is a function of the brightness and velocity distributions independent from the local line profile. This property of DI has been investigated for rotating stars, theoretically by Chelli \& Petrov (1995b) and experimentally on the $\mathrm{K}$ giant Aldebaran (Petrov et al, 1996) were it permitted to measure the angular diameter $(20.5+1$ milliarseconds $)$ and the equatorial rotation velocity $(5.8+1 \mathrm{~km} / \mathrm{s})$ although both were under telescope ( 80 milliarseconds) and spectrograph $(17 \mathrm{~km} / \mathrm{s})$ resolution limit. The possibility to eliminate $p(\lambda)$ makes it possible to use complex part of the spectrum with groups of more or less blended lines without necessity to carefully model it. Another very interesting aspect is that even if the local profiles emitted by various parts of the source are different, they are all convoluted by the same spectral point spread function $g(\lambda)$ and Eq. (10) shows that $\varphi(\sigma)$ is intrinsically independent from any fluctuation of the instrumental profile, just as $\phi\left(\vec{u}, \lambda_{1}, \lambda_{r}\right)$ is insensitive to any variation of the atmospheric turbulence. This built-in stability can be applied for example to astroseismology.

\section{Application of Differential Interferometry to Doppler Imaging}

\subsection{PHOTOCENTER DISPLACEMENTS FOR A ROTATING STAR}

Figure 1 describes the $\vec{\varepsilon}(\lambda)$ curves which can be expected from a spotted star rotating as a solid body and shows their variations through a period of rotation. The computation was made for $V \sin i=30 \mathrm{~km} / \mathrm{s}$ and a $p(\lambda)$ with FWHM $=10 \mathrm{~km} / \mathrm{s}$. Let $\varepsilon_{x}(\lambda)$ and $\varepsilon_{y}(\lambda)$ be the components of $\vec{\varepsilon}(\lambda)$ in the $\mathrm{x}$ and $\mathrm{y}$ axes, the second being the rotion axis. 


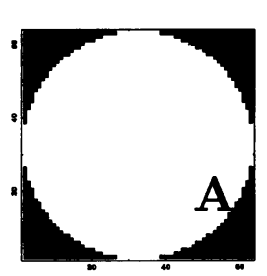

$\mathbf{s}(\lambda)$

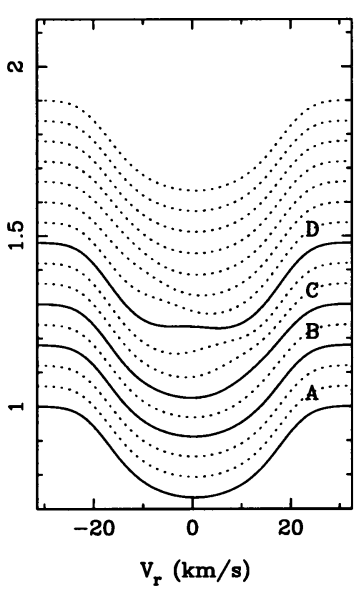

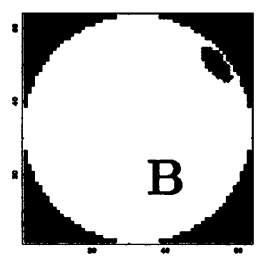
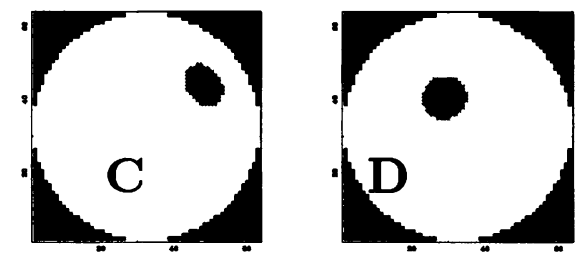

$\epsilon_{\mathbf{x}}(\lambda)$

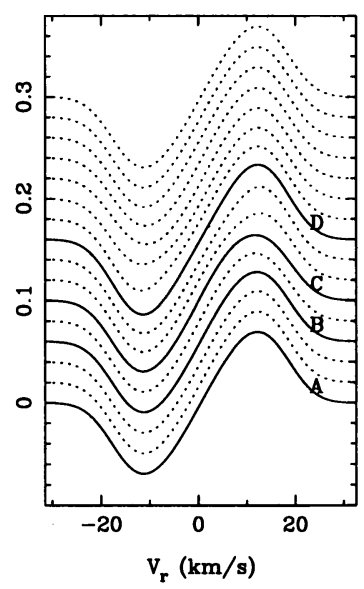

$\epsilon_{\mathbf{y}}(\lambda)$

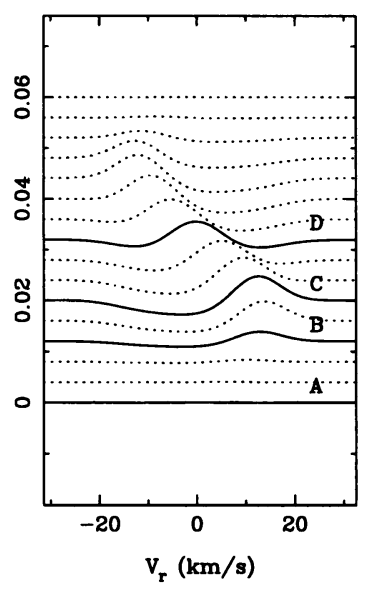

Figure 1. Spectrum and photocenter displacement for a star at different phases of rotation. $s(\lambda)$ represents the normalised flux. $\varepsilon_{\mathbf{x}}(\lambda)$ and $\varepsilon_{\mathbf{y}}(\lambda)$ are the components of the displacement respectively perpendicular and parallel to the stellar rotation axis. They are given in stellar diameter units. The scales are correct only for the lower curves in each figure, the other one being incremented to display the evolution throughout one period. We assumed Vsini $=30 \mathrm{~km} / \mathrm{s}$ and a local profile of $10 \mathrm{~km} / \mathrm{s}$ FWHM. The spot FWHM is 0.2 stellar diameter. The full lines labelled A, B, C, D, correspond to the stellar images designed by the same letters

First we consider the case on a non spotted stellar disk, such as the one represented by the image and curves (A). When we observe in a narrow band in the wing of a rotation widened absorption line, we are actually looking at a disk with a darkened strip parallel to the rotation axis where the radial velocity is close to what is needed to center the line in the observation channel. The photocenter of this image is moved away from the darkened zone. The effects have opposite signs in the two line wings. They are small in the extreme wings of the line e.g. $= \pm 20 \mathrm{~km} / \mathrm{s}$ ), when the darkened area is a small strip near an edge of the star. They culminate somewhere in the middle of the wings (here $\pm 10 \mathrm{~km} / \mathrm{s}$ ) when the darkened area is large but remains far from the axis and go to zero in the center of the line because a centered darkened strip does not introduce any asymmetry. For 
a non spotted stars, the photocenter displacements are perpendicular to the rotation axis and $\varepsilon_{y}(\lambda)=0$. The maximum amplitude of $\varepsilon_{x}(\lambda)$, obtained when Vsini is close to the FWHM of the local profile is order of 0.12 stellar diameters.

A spot on the stellar disk, like in figure and curves (B), results in the apparition of a bump in the absorption line profile and in the $\varepsilon_{x}(\lambda)$ curve. Instantaneous measures of $s(\lambda)$ and of $\varepsilon_{x}(\lambda)$ do not contain information about the y coordinates of the spots but $\varepsilon_{y}(\lambda)$ displays a relatively sharp shift toward the spot of a quantity related to its y coordinate. For a small spot of equivalent area $a_{s}$ on a stellar disk of area $a_{d}$ the maximum $\varepsilon_{y}(\lambda)$ is

$$
\max \left[\varepsilon_{y}(\lambda)\right]=y \frac{a_{s}}{a_{d}} .
$$

\subsection{POTENTIALITIES OF DIFFERENTIAL INTERFEROMETRY IMAGING}

The most obvious advantage of applying DI to Doppler Imaging is that it eliminates the north south ambiguity and the associated mirror effect on close to equator on stars. Petrov (1988) confirmed this with computer simulations of a complete image reconstruction. More generally, DI multiplies by 3 the number of independent equations relating the measures and the image and we have good reasons to believe that this could be a dramatic improvement each time Doppler Imaging is used close to its limits.

Another property tested with computer simulations is that velocity and brightness features with extremely similar spectral signatures can have a completely different effect on $\varepsilon_{y}(\lambda)$. This offers us a way to help decide if the periodic variability of $\mathrm{Be}$ stars is due to non radial pulsation or to rotational modulation (Baade et Balona 1993). More generally, DI might help constrain the radial velocity field. For example differential rotation will break the equivalence between $s(\lambda)$ and $\varepsilon_{x}(\lambda)$ existing for solid rotation and this should offer a direct way to measure it.

If we have an estimation of the sizes and contrast of spots, for example from photometry, the latitude of a relatively isolated structure can be estimated from instantaneous $\varepsilon_{y}(\lambda)$ measures. This can help monitoring spots whose stellar coordinate change significantly during one rotation period.

We saw how a local line profile independent from space can be eliminated. This should get rid of the very strong sensitivity of reconstructed images to inperfectly deblended lines underlined by Unruh (1996). We believe that this can be generalised to profiles with known latitudinal and temperature dependence. Polar caps should have a specific signature on $\varepsilon_{y}(\lambda)$, similar to curve (D) in figure 1 but invariable in time. Observing it would help confirming that they are not due to errors in the local profiles. 


\section{Estimation of the signal to noise ratios}

Combining efficiently $s(\lambda)$ and $\vec{\varepsilon}(\lambda)$ assumes that they have comparable SNRs. If $n(\lambda)$ is the total number of photons collected in the channel $\lambda$, the uncertainties on $s(\lambda)$ and $\vec{\varepsilon}(\lambda)$ are given by:

$$
\begin{gathered}
\sigma_{s(\lambda)}=n(\lambda)^{-1 / 2} \\
\sigma_{\varepsilon(\lambda)}=\sup (\phi, \lambda / B) n(\lambda)^{-1 / 2}
\end{gathered}
$$

where $\phi$ is the stellar diameter and $\lambda / B$ is the diffraction resolution limit for a baseline $B$ (for a single aperture telescope $B$ is equal to the diameter). Eq. (13) gives the error on the photodisplacement due to photon noise for a bright star (Petrov 1989). Lagarde (1994) demonstrated that it is possible to reach this limit even for a magnitude 0 star observed with a relatively poor detector. Bright star means that the number $p_{r}$ of photons per speckle and per image in the reference channel is larger than 1 . The limiting magnitude for this condition ranges from 5 to 8 as a function of seeing and experimental set up. For fainter stars, $\sigma_{\varepsilon(\lambda)}$ must be multiplied by $p_{r}^{1 / 2}$. Using the same notations and assumptions than for Eq. (11) the maximum spectrum and displacement perturbations are

$$
\Delta s_{\max }=\frac{a_{s}}{a_{d}}, \quad \Delta \varepsilon_{\max }=\frac{\phi}{2} \frac{a_{s}}{a_{d}}
$$

and combining Eqs. (12), (13) and (14), yields the ratio between the SNRs of DI and Spectroscopy

$$
\frac{\operatorname{SNR}(\mathrm{DI})}{\operatorname{SNR}(\operatorname{Spectroscopy})}=\frac{\Delta \varepsilon_{\max } / \sigma_{\varepsilon(\lambda)}}{\Delta s_{\max } / \sigma_{s(\lambda)}}=\frac{\phi / 2}{\sup (\phi, \lambda / B)}
$$

If we are observing a 5 milliarcseconds star (e.g. magnitude 3.5 G8III star) with a $4 \mathrm{~m}$ telescop, the DI SNR will be about 5 times lower than the spectroscopic one which is probably prohibitive except for the very brightest candidates. But with a $20 \mathrm{~m}$ baseline interferometer DI SNR can approach half of the spectroscopic SNR. With the $\sim 100 \mathrm{~m}$ baseline large telescope interferometers which will be available soon, this condition can be realised on a fair number of stars (up to magnitude 7 or 8 for $\mathrm{K}$ giants for example).

\section{Conclusions}

We have presented some of the characteristics of DI which can contribute to better Doppler Images. Except for the obvious elimination of the north- 
south ambiguity, what we have is much closer to a list of points to investigate than to a catalogue of solutions but we are confident that real progress will be obtained as soon as we will have DI data with a quality similar to what is used in spectroscopy. This needs a resolution limit close to the stellar angular diameter. With the $\sim 100 \mathrm{~m}$ baseline interferometers with large telescope which will be available soon, this will be possible on a fair number of stars. Then, Differential Interferometry Imaging (DII) is likely to be able to analyse stellar surface structures whose direct imaging would require kilometric baselines. The condition is to have relatively high resolution spectrographs at the coherent focus of this interferometers. This will be partially the case at the GI2T interferometer in southern France in 1997 when the automatic Fringe Sensor Unit developed for ESO will make it possible to use the high spectral resolution $(30000)$ mode of the new focal instrument. But a full exploitation of the potential of DII will need a dedicated Echelle instrument installed at the focus of a three telescopes interferometer.

Acknowledgements. R. G. Petrov thanks the "Departement Fresnel" of the "Observatoire de la Cote d'Azur" for the financial support to his travel to Vienna.

\section{References}

Aime, C., Kadiri, S., Martin, F., Petrov, R.G., Ricort, G. (1984) $A \& A, 134,354$.

Baade, D., Balona, L.A. (1994) Pulsation, Rotation and Mass Loss in Early-Type stars, Proc. IAU Colloq. 162, Balona L. A. et al. (eds), p. 311.

Beckers, J.M. (1982) Cpt. Acta, 29, 361.

Chelli, A., Petrov, R.G. (1995) $A \mathscr{E} A S, \mathbf{1 0 9}, 389$.

Chelli, A., Petrov, R.G. (1995) $A \& A S$, 109, 401.

Lagarde, S. (1994) Traitement des donnees et premiers resultats experimentaux en interferometrie differentielle des tavelures, Doctoral thesis, University of Nice.

Petrov, R.G., Lagarde, S., Blazit, A., Foy, R., Martin, F. (1996) $A \mho A A$, submitted

Petrov, R.G., Balega, Y.Y., Blazit, A., Vassyuk, V.V., Lagarde, S., Foy, R. (1995) Astron. Letters., in press

Petrov, R.G. (1989) Diffraction limited imaging with very large telescopes, Alloin, D. and Mariotti, J.M. (eds), NATO ASI series C 274, p. 249.

Petrov, R.G., Roddier F., Aime C. (1986) J. Cpt. Soc. Am. A, 3, p. 634.

Rice, J.B. (1996) Stellar Surface Structures, Proc. IAU Symp. 176, Strassmeier K. G. and Linsky J. L. (eds), this volume.

Strassmeier K. G. (1996) Stellar Surface Structures, Proc. IAU Symp. 176, Strassmeier K. G. and Linsky J. L. (eds), this volume.

Unruh, Y. (1996) Stellar Surface Structures, Proc. IAU Symp. 176, Strassmeier K. G. and Linsky J. L. (eds), this volume. 


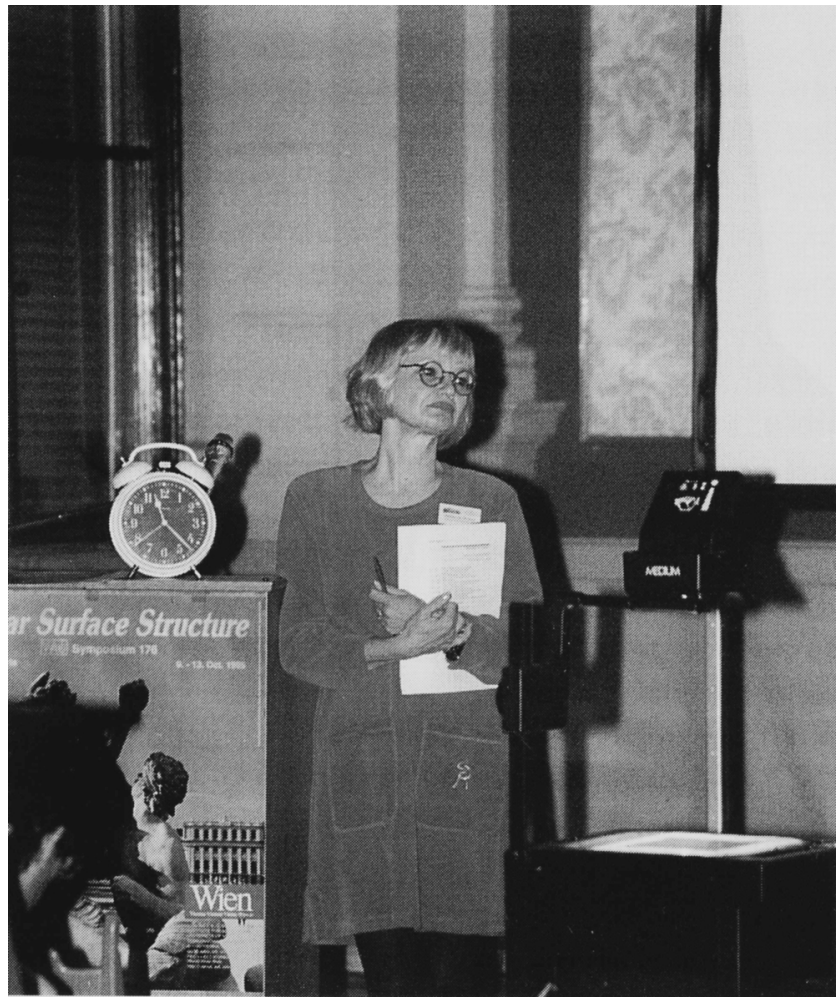

Session chair Andrea Dupree tried to keep the speakers within their alloted time

John Baldwin (bottom) found that very amusing.

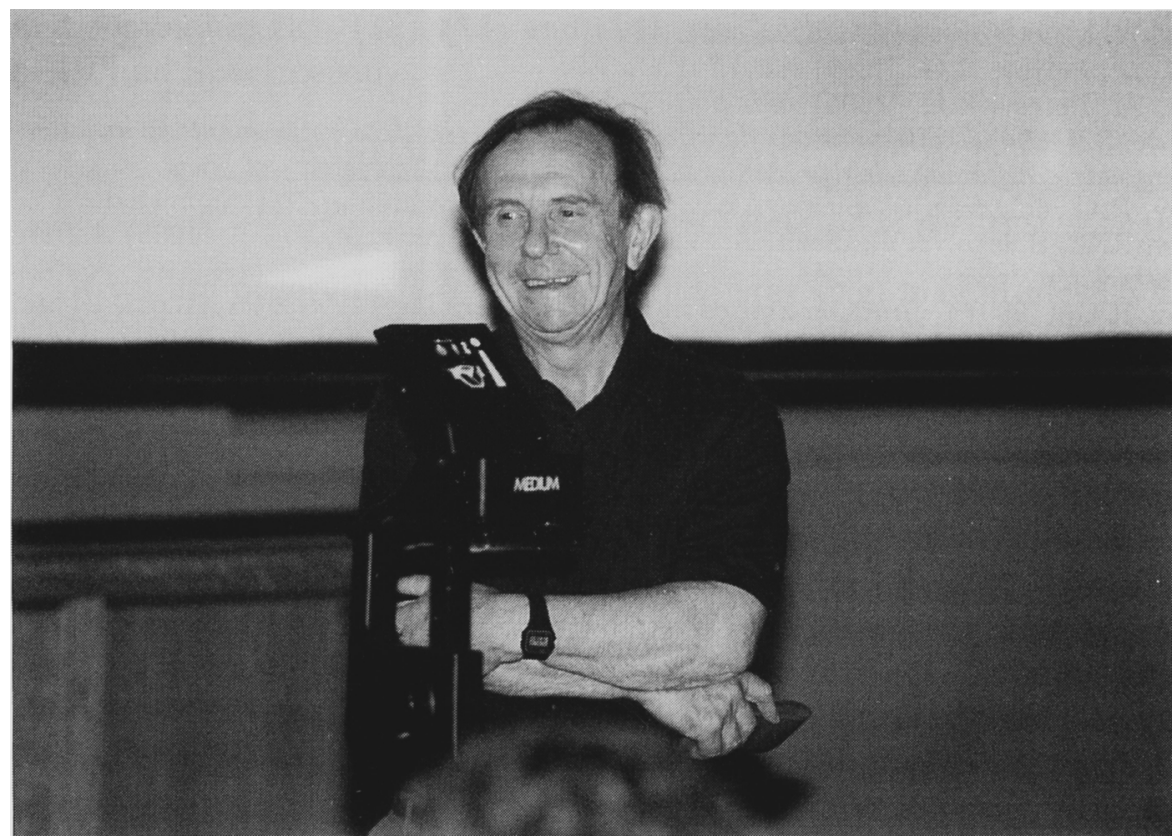

